\title{
Thermal diffusivity measurement of silicon samples by a combined piezoelectric and pyroelectric method
}

\author{
M. Aravind and P. C. W. Fung \\ Department of Physics, The University of Hong Kong, Hong Kong
}

(Received 29 October 1998; accepted for publication 15 September 1999)

\begin{abstract}
In the past, when thermal diffusivity measurement of materials were carried out by photoacoustic signal detection using transducers, only the piezoelectric or the pyroelectric property of the transducers was considered. In case the transducer exhibits both piezoelectric and pyroelectric properties, one of these properties had been suppressed during the experimentation, obviously more errors are introduced this way. We use polyvinylidene difluoride (PVDF) as the detector for thermal waves. Since PVDF has both piezoelectric and pyroelectric properties, we show in this article that the signal detected by the transducer is a sum of both the piezoelectric and pyroelectric effects. Silicon semiconductor samples are considered in this article to compare the theory with experimental results. Although both the piezoelectric and pyroelectric properties are found in the resultant signal at all the frequency ranges considered, we find that when the samples are thermally thick, the piezoelectric contribution to the detected signal is slightly more than the pyroelectric contribution and vice versa when the sample is thermally thin. This behavior of the combined signal can be explained by the fact that in an optically opaque solid heat is generated very close to the surface, following absorption. This heat is communicated to the PVDF as long as the thermal diffusion length is larger than the thickness (i.e., the sample is thermally thin). At high frequencies the solid becomes thermally thick and the pyroelectric nature decreases as both the optical and thermal contact of the sample with the detector diminishes. Since both the properties are considered in our theory, we can measure the thermal diffusivity of a general sample without "artificial suppression." Moreover, from our analysis we can deduce the physical thickness of the sample from the critical frequency, which is the frequency at which the sample changes from thermally thin to thermally thick. This transition is clearly evident in the amplitude curve as a change in slope is detected at the critical frequency. (C) 1999 American Institute of Physics.

[S0034-6748(99)04112-X]
\end{abstract}

\section{INTRODUCTION}

Photoacoustic microscopy ${ }^{1}$ has been used as a nondestructive evaluation method for determining crucial thermal properties of different kinds of materials. Various photoacoustic methods like the two-beam phase lag, ${ }^{2}$ mirage technique, ${ }^{3}$ piezoelectric detection, ${ }^{4,5}$ pyroelectric detection, ${ }^{6}$ etc., are used in the measurement of thermal diffusivity of materials. In the past, either the pyroelectric or the piezoelectric effect of the transducer was utilized during the measurement of thermal diffusivity. In this article we chose polyvinylidene diflouride (PVDF) as the transducer which demonstrates both piezoelectric and pyroelectric properties. Previously, when PVDF was considered as a transducer. Christofides et $\mathrm{al}^{7}{ }^{7}$ reduced the piezoelectric nature of PVDF at high frequencies by coating the sample surfaces with black paint, thus maximizing the thermal contribution and minimizing the direct transmission signal. We extend the existing theory on thermal diffusivity to incorporate both the piezoelectric and pyroelectric effects. A direct comparison between the theory and experimental results is also made. During experimentation the PVDF transducer is directly attached to the sample by a little vacuum grease in gel form.

Silicon semiconductors of various thickness have been chosen as our samples because of their wide use as electronic and optical materials. Power dissipation in microelectronic and optoelectronic devices is an important mechanism in understanding the device performance. The room-temperature value of thermal diffusivity of the materials used in these devices is an important parameter in optimizing the device. Thermal diffusivity $(\alpha)$ determines the rate of diffusion of heat in a material. Apart from its own intrinsic importance, its determination gives the value of thermal conductivity $\kappa$, if the density $\rho$ and thermal capacity at constant pressure $C$ are determined, since $\alpha=\kappa / \rho C 8^{8}$ (a well-known expression).

In this article, we are considering a theory to incorporate the effects of both piezoelectric and pyroelectric properties present in the transducer. The equations obtained theoretically for the amplitude and phase of the signal [Eqs. (11) and (12)] were fitted to the experimentally observed amplitude and phase of the signal, from the lock-in amplifier, as a function of the chopping frequency. In this fitting, the adjusted parameter is the thermal diffusivity of the sample. Thus, the thermal diffusivity value is obtained as a fitting parameter from the phase and amplitude graphs of the signal. The thicknesses of the samples considered are such that they are not thermally thick at the low-frequency range of our experiment. The transition from the thermally thin to thermally thick region is clearly evident from the graphs. Determining $f_{c}$ - the critical frequency at which the transition occursfrom the graph, the thickness of the samples whose ther- 
mal diffusivity is known can also be calculated since $l$ $=\sqrt{\alpha / \pi f_{c}}$.

\section{THEORY}

In the piezoelectric detection technique, the stresses and strains developed in the sample as a result of heat absorption are converted to a measurable electric voltage by the transducer. A pyroelectric material is one which possesses an inherent electrical polarization, the magnitude of which is a function of temperature. A change in temperature in the sample due to modulated light absorption, manifests itself as a potential difference between the upper and lower surfaces of the pyroelectric transducer which is in intimate contact with the sample. This is the basis of the pyroelectric detection.

Piezoelectric and pyroelectric properties are present in the polymer PVDF. These properties are due to the combination of electrical, mechanical, and acoustic properties of this polymer. The piezoelectric and pyroelectric effects are described in terms of compliances between electric polarization $(P)$ and stress $(S)$ or temperature $(T)$, respectively, giving the piezoelectric constant $(e)$ and the pyroelectric constant $(p)$. The electric polarization is related to the electric displacement $(D)$ by the relation ${ }^{9}$

$$
D=\epsilon_{0} E+P,
$$

where $\epsilon_{0}$ is the dielectric constant.

The constitutive relations of piezoelectric crystals provide the coupling between the mechanical and electrical fields. The polymer PVDF possesses some spontaneous polarization. If this polarization is changed from its original value by an applied strain or temperature variation, the film exhibits piezoelectricity or pyroelectricity, respectively. The primary pyroelectric coefficient is measured when the crystal volume and shape are clamped. When the sample is free to expand or contract, the resultant strain causes a piezoelectrically induced polarization which must be added to the pyroelectric effect.

The equilibrium properties of crystals are defined using thermodynamic theory. The thermodynamic variables are stress $(S)$, strain $(\tau)$, electric field $(E)$, electric displacement $(D)$, temperature $(T)$, and entropy $(H)$. For a change of independent variables $(S, E, T)$ the corresponding equations of state of the dependent variables $(\tau, D, H)$ are given by ${ }^{9}$

$$
\begin{aligned}
& \tau=c^{E} S+e^{T} E+\gamma^{E} T, \\
& D=e^{T} S+\epsilon^{S T} E+p^{S} T, \\
& H=\gamma^{E} S+p^{S} E+C^{E S} T / T_{0} .
\end{aligned}
$$

$c^{E}, e, \gamma, \epsilon, p$, and $C$ are the elastic compliance tensor, piezoelectric stress tensor, thermal expansion tensor, dielectric tensor at constant stress, pyroelectric vector, and the volume specific heat, respectively. $T_{0}$ is the ambient temperature. The superscripts denote the variables held constant when the property is measured.

Substituting the value of stress in Eq. (2a) into Eq. (2b), we get the electric displacement as

$$
D=d^{T} \tau+\epsilon^{\tau T} E+p^{\tau} T,
$$

where $\quad d^{T}=e^{T} / c^{E}, \quad \epsilon^{\tau T}=\epsilon^{S T}-\left(e^{T}\right)^{2} / c^{E}, \quad$ and $\quad p^{\tau}=p^{S}$ $-\gamma^{E} e^{T} / c^{E}$, which are the piezoelectric strain tensor, dielectric tensor at constant strain, and the pyroelectric vector at constant strain, respectively.

Let " $u$ " be the infinitesimal mechanical displacement of a material point in the transducer. This is the same as the displacement in the sample, when the thickness of the transducer is assumed to be small as compared to the thickness of the sample. The spatial gradient of the mechanical displacement determines the strain tensor:

$$
\tau_{i j}=\frac{\partial u_{i}}{\partial x_{j}} .
$$

The transducer is fixed to a rigid backing so that at $z=-l$ there is no displacement in the transducer. It is also assumed that there is no stress at $z=0$, i.e., the sample is free to expand with the transducer minimally impeding its motion.

When we consider the case of no electrical displacement within the crystal, i.e., $D=0$, then from Eqs. (3) and (4),

$$
E=-\frac{d^{T}}{\epsilon^{\tau T}} \frac{d u}{d x}-\frac{p^{\tau}}{\epsilon^{\tau T}} T .
$$

The potential difference between the electrodes of the transducer is obtained as

$V=-\int_{-l}^{0} E d x=\frac{d^{T}}{\epsilon^{\tau T}} \int_{-l}^{0} \frac{d u}{d x} d x+\frac{p^{\tau}}{\epsilon^{\tau T}} \int_{-l}^{0} T d x=V_{\mathrm{pz}}+V_{\mathrm{py}}$.

Thus, we see that the resultant voltage is the sum of the piezoelectric and pyroelectric effect.

$V_{\mathrm{pz}}$ for an optically opaque sample is obtained $\mathrm{as}^{4}$

$$
V_{\mathrm{pz}}=-\frac{4 X l_{p}}{k_{s} l_{s} \sigma_{s}^{2} S_{p}}\left(1+\frac{3\left(1-\cosh \left(\sigma_{s} l_{s}\right)\right)}{\sigma_{s} l_{s} \sinh \left(\sigma_{s} l_{s}\right)}\right)=R_{\mathrm{pz}}+i I_{\mathrm{pz}},
$$

where $X=I \pi b^{2} \gamma e / \epsilon$ and $\sigma_{s}=(1+i) a_{s} . I, b, \gamma, e, \epsilon$, and $S_{p}$ are the incident light intensity, the beam radius, thermal expansion coefficient, piezoelectric stress tensor, dielectric constant, and the surface area of the transducer, respectively. $l$, $k$, and $a$ are, respectively, thickness, thermal conductivity, and thermal diffusion coefficient of a substance. The subscripts $s, p$, and $g$ denote sample, transducer, and gas, respectively. The amplitude of the piezoelectric part of the signal can now be obtained as

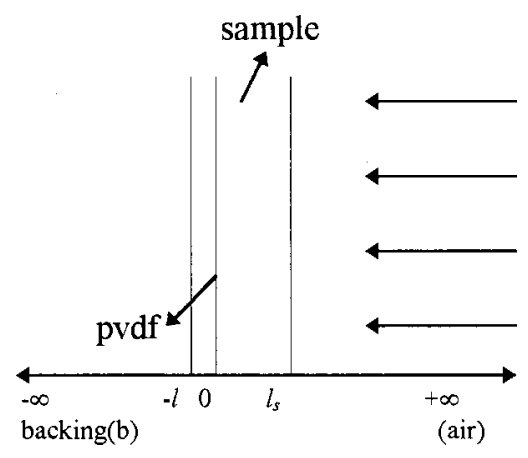

FIG. 1. The figure gives the schematic of the experimental setup. 


$$
A_{\mathrm{pz}}=\frac{X l_{p}}{\left(a_{s} l_{s}\right)^{2} S_{p}} \frac{1}{k_{s} a_{s}} \frac{\left\{\left[2 a_{s} l_{s}\left(\cosh \left(a_{s} l_{s}\right)+\cos \left(a_{s} l_{s}\right)\right)-3\left(\sinh \left(a_{s} l_{s}\right)+\sin \left(a_{s} l_{s}\right)\right)\right]^{2}+\left[3\left(\sinh \left(a_{s} l_{s}\right)-\sin \left(a_{s} l_{s}\right)\right)\right]^{2}\right\}^{1 / 2}}{\cosh \left(a_{s} l_{s}\right)+\cos \left(a_{s} l_{s}\right)} .
$$

Considering an optically opaque and thermally thick transducer and an optically opaque sample with $r_{s} \gg 1$, the pyroelectric voltage is obtained from Ref. 6 as

$$
V_{\mathrm{py}}=\frac{Y}{2 k_{p} \sigma_{p}^{2}} \frac{b_{p s}}{1+b_{g p}} \frac{1}{b_{p s} \cosh \left(\sigma_{s} l_{s}\right)+\sinh \left(\sigma_{s} l_{s}\right)}=R_{\mathrm{py}}+i I_{\mathrm{py}}
$$

where $Y=I_{p} / \epsilon \epsilon_{0}, b_{p s}=k_{p} a_{p} / k_{s} a_{s}$, and $b_{g p}=k_{g} a_{g} / k_{p} a_{p} . p, \epsilon$, and $\epsilon_{0}$ are the pyroelectric vector, dielectric constant, and the permittivity of the transducer, respectively. The amplitude of the pyroelectric part of the signal can now be obtained as

$$
A_{\mathrm{py}}=\frac{Y}{4 a_{p}} \frac{1}{k_{s} a_{s}} \frac{\left\{\left[\left(b_{p s} \sinh \left(a_{s} l_{s}\right)+\cosh \left(a_{s} l_{s}\right)\right) \sin \left(a_{s} l_{s}\right)\right]^{2}+\left[\left(b_{p s} \cosh \left(a_{s} l_{s}\right)+\sinh \left(a_{s} l_{s}\right)\right) \cos \left(a_{s} l_{s}\right)\right]^{2}\right\}^{1 / 2}}{\left(b_{p s} \sinh \left(a_{s} l_{s}\right)+\cosh \left(a_{s} l_{s}\right)\right)^{2}+\left(b_{p s}^{2}-1\right) \cos ^{2}\left(a_{s} l_{s}\right)} .
$$

As all the samples considered are optically thick, there is no reflection of the incident radiation back into the sample from the surface of the transducer. The output signal is assumed to be due to the sum of the piezoelectric and pyroelectric contributions in the transducer. Hence, the amplitude of the total signal can be written as

$$
A_{\text {tot }}=\left\{\left(R_{\mathrm{pz}}+R_{\mathrm{py}}\right)^{2}+\left(I_{\mathrm{pz}}+I_{\mathrm{py}}\right)^{2}\right\}^{1 / 2},
$$

and the phase of the signal can be expressed as

$$
P_{\mathrm{tot}}=\tan ^{-1}\left(\frac{I_{\mathrm{pz}}+I_{\mathrm{py}}}{R_{\mathrm{pz}}+R_{\mathrm{py}}}\right)
$$

where

$$
\begin{aligned}
R_{\mathrm{pz}} & =\frac{X l_{p}}{\left(a_{s} l_{s}\right)^{2} S_{p}} \frac{1}{k_{s} a_{s}} \frac{\left[2 a_{s} l_{s}\left(\cosh \left(a_{s} l_{s}\right)+\cos \left(a_{s} l_{s}\right)\right)-3\left(\sinh \left(a_{s} l_{s}\right)+\sin \left(a_{s} l_{s}\right)\right)\right]}{\cosh \left(a_{s} l_{s}\right)+\cos \left(a_{s} l_{s}\right)}, \\
I_{\mathrm{pz}} & =\frac{X l_{p}}{\left(a_{s} l_{s}\right)^{2} S_{p}} \frac{1}{k_{s} a_{s}} \frac{\left[3\left(\sinh \left(a_{s} l_{s}\right)-\sin \left(a_{s} l_{s}\right)\right)\right]}{\cosh \left(a_{s} l_{s}\right)+\cos \left(a_{s} l_{s}\right)}, \\
R_{\mathrm{py}} & =\frac{-Y}{4 a_{p}} \frac{1}{k_{s} a_{s}} \frac{\left[\left(b_{p s} \sinh \left(a_{s} l_{s}\right)+\cosh \left(a_{s} l_{s}\right)\right) \sin \left(a_{s} l_{s}\right)\right]}{\left(b_{p s} \sinh \left(a_{s} l_{s}\right)+\cosh \left(a_{s} l_{s}\right)\right)^{2}+\left(b_{p s}^{2}-1\right) \cos ^{2}\left(a_{s} l_{s}\right)}, \\
I_{\mathrm{py}} & =\frac{-Y}{4 a_{p}} \frac{1}{k_{s} a_{s}} \frac{\left[\left(b_{p s} \cosh \left(a_{s} l_{s}\right)+\sinh \left(a_{s} l_{s}\right)\right) \cos \left(a_{s} l_{s}\right)\right]}{\left(b_{p s} \sinh \left(a_{s} l_{s}\right)+\cosh \left(a_{s} l_{s}\right)\right)^{2}+\left(b_{p s}^{2}-1\right) \cos ^{2}\left(a_{s} l_{s}\right)} .
\end{aligned}
$$

\section{EXPERIMENTAL SETUP}

The experimental setup and the schematic are shown in Figs. 1 and 2, respectively. A Coherent-Innova 70, Argonion laser (wavelength $5145 \AA$, output power $6 \mathrm{~W}$ ) was used as the light source. Periodic heating of the sample was achieved by chopping the laser beam using a Stanford SR 540 mechanical chopper, which is computer controlled. The signal from the transducer is detected by a Stanford SR 530 lock-in amplifier. The phase of the signal was compared with that of the reference signal from the chopper. All measurements were performed on a Newport RS4000 optical table to minimize spurious signals due to unwanted vibration.

The laser beam heats the front surface of the sample. The stress and strain arising in the sample due to the increase in temperature on the surface of the sample as well as the temperature difference between the sample surface and the transducer surface is detected by the PVDF transducer attached to the rear surface of the sample with a little vacuum grease. This signal is amplified using a preamplifier and is then fed to the lock-in amplifier for phase and amplitude measurement. The lock-in amplifier is also controlled using the computer. All the measurements are done at room temperature.

\section{RESULTS AND DISCUSSION}

The theoretical and experimental results for the amplitude and the phase of the signal are shown in Figs. 3 and 4,

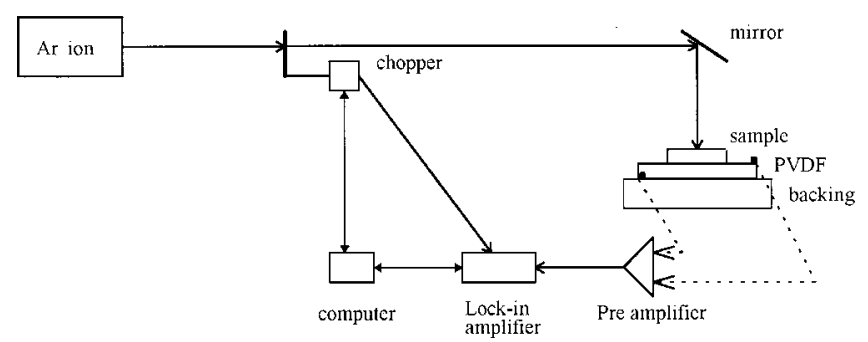

FIG. 2. Experimental setup. 


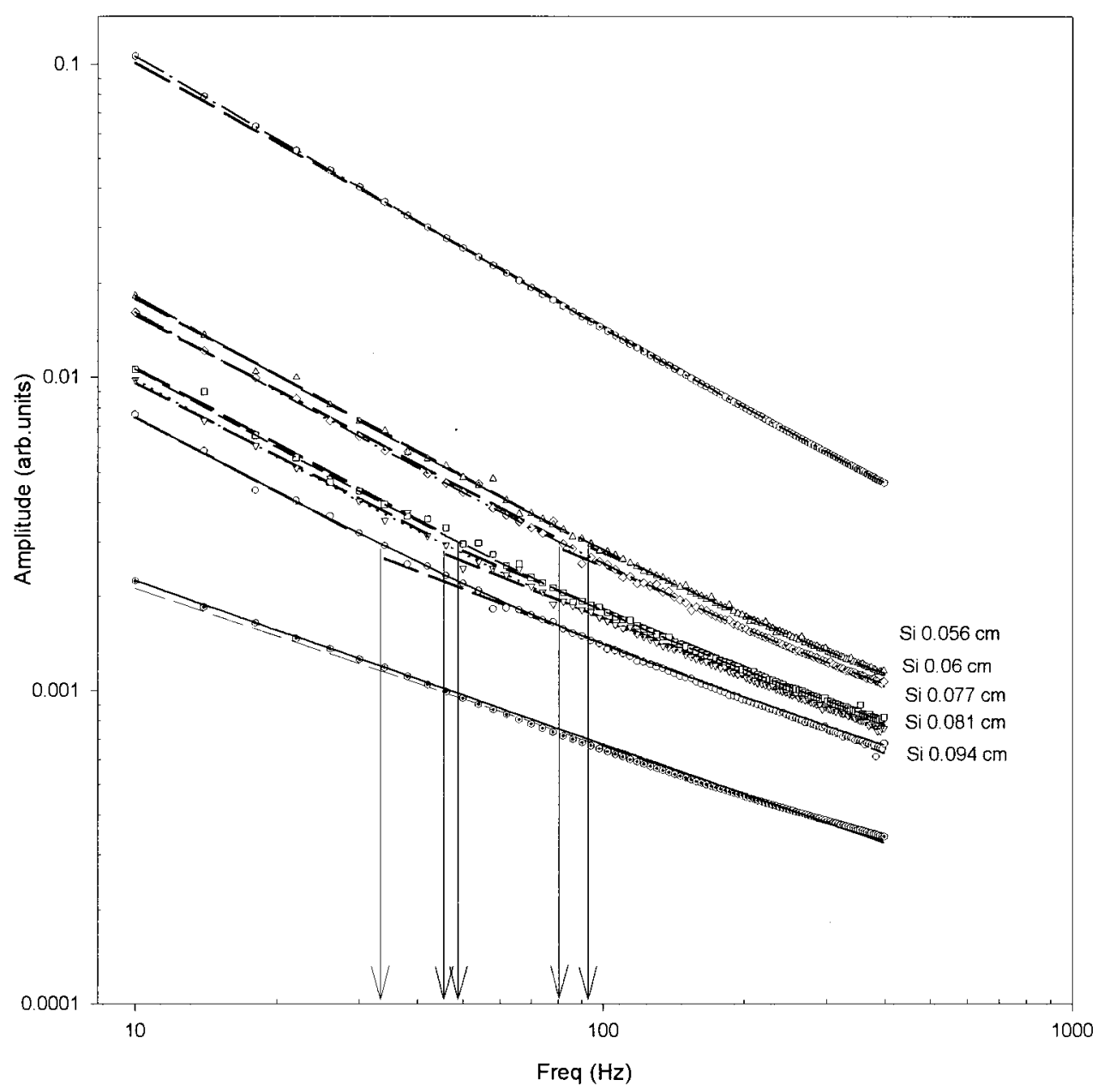

FIG. 3. Experimentally obtained amplitude as a function of the chopping frequency and the theoretical fitting (continuous line) for silicon samples of various thickness. The thicker dashed line along the amplitude plot shows the change in slope at the critical frequency. The upper and lower curves are theoretically obtained amplitude values for samples which may be thermally thin or thermally thick, respectively, in the entire frequency range considered.

respectively. The continuous curve in Figs. 3 and 4 shows the calculated amplitude and phase by using the thermal diffusivity value of the sample as the fitting parameter in Eqs. (11) and (12), respectively. The average thermal diffusivity of the silicon samples thus determined is 0.8973 $\pm 0.0104 \mathrm{~cm}^{2} / \mathrm{s}$, which compares well with the value of silicon obtained previously by other authors. ${ }^{10,11}$

Consider the silicon sample of thickness $0.056 \mathrm{~cm}$. For a comparison of the effect of the pyroelectric and piezoelectric nature, it is shown in Fig. 5. We take the thermal diffusivity value of silicon to be $0.8973 \mathrm{~cm}^{2} / \mathrm{s}$. The critical frequency $f_{c}$ of the sample is then obtained as $91 \mathrm{~Hz}$ (at $f_{c}$, the thermal diffusion length of the sample becomes equal to its physical thickness). It is the frequency at which the sample changes from thermally thin to thermally thick. From Fig. 5 we can see a change in slope in the amplitude curve around $91 \mathrm{~Hz}$. Thus, we see that a change of slope in the amplitude curve will predict the phase change of the sample from thermally thin to thermally thick. This shows that if we have a sample of unknown thickness, the determination of the critical frequency from the graph can give the thickness of the sample if the thermal diffusivity is known, since $l_{s}=\sqrt{\alpha / \pi f_{c}}$.

From Fig. 5, we can see that at the critical frequency there is a crossover between the purely piezoelectric (dark circles) and purely pyroelectric (white circles) amplitudes as calculated using Eqs. (9) and (10), respectively. We see that below the critical frequency the pyroelectric nature is predominant in the signal and above which the piezoelectric nature is predominant. Hence, neither the piezoelectric nor the pyroelectric effect alone can represent the output signal (squares) from the lock-in amplifier.

The thickness of the PVDF transducer used in the experiment is $0.0054 \mathrm{~cm}$. Hence, the transducer is thermally thick at all frequencies, as the thermal diffusivity of PVDF is $0.00054 \mathrm{~cm}^{2} / \mathrm{s}^{12}$

In Table I we give the mean difference, which is the difference between experimentally obtained and theoretically calculated values of the amplitude/phase. In calculating the amplitude and phase, the thermal diffusivity value is used as the fitting parameter. The thermal diffusivity values thus obtained from the amplitude curve and phase curve are also given in Table I. The average value of the thermal diffusivity obtained is $0.8973 \pm 0.0104 \mathrm{~cm}^{2} / \mathrm{s}$. The mean difference and the resultant thermal diffusivity obtained show that the theory developed here is quite accurate in determining the thermal diffusivity of general solid samples.

Previously, in experiments to measure thermal diffusivity of samples by photoacoustic methods using the transducer as the signal detector, either the piezoelectric or the pyroelectric property of the transducer was considered. In 


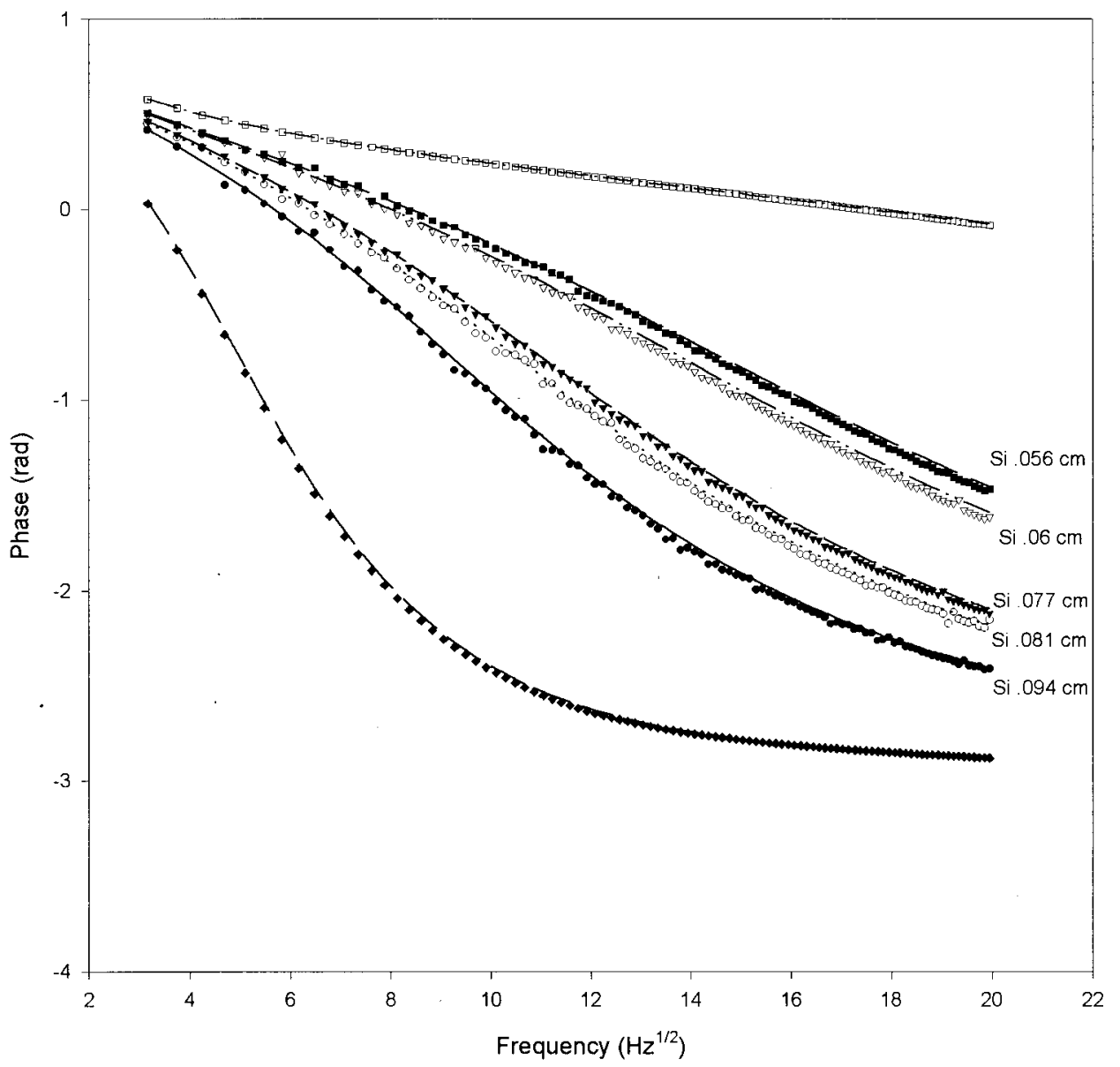

FIG. 4. Experimentally obtained phase signal as a function of the chopping frequency and the theoretical fitting (continuous line) for silicon samples of various thickness. The upper and lower curves are theoretically obtained phase values for samples which may be thermally thin or thermally thick, respectively, in the entire frequency range considered.

case the transducer exhibited both the properties, either the piezoelectric or the pyroelectric nature of the transducer was purposely suppressed during the experimentation. Several authors have used $Z$-cut ${ }^{13,14} \mathrm{LiTaO}_{3}$ as the pyroelectric transducer. It has been shown that the pyroelectric effect contributed predominantly to the PA signals for the Z-cut plate. Christofides ${ }^{7}$ et al. have reduced the piezoelectric behavior of the PVDF transducer at high frequencies by coating the sample surfaces with black paint.

In the article by Blonskij ${ }^{4}$ to determine the thermal diffusivity of a sample using lead zirconate titanate (PZT) as the transducer, it can be seen that the calculated amplitude does not match the observed values in the low-frequency range. We believe that is due to not considering the pyroelectric effect of the signal from the transducer at low frequencies.

Our method thus provides an effective means of calculating the thermal diffusivity of solids by taking into account both the piezoelectric and pyroelectric nature of the transducer. The experimental setup is very simple as shown in Fig. 1. The method is very sensitive to phase transitions as can be seen from the amplitude and phase graphs. The critical frequency at which the sample moves from the thermally thin to thick region is clearly evident in the amplitude curve. Hence, we believe this methodology of combining the piezoelectric and pyroelectric nature of the signal from a PVDF

TABLE I. The mean difference, which is the average of the difference between experimentally obtained and theoretically calculated values in the amplitude/ phase is given. Thermal diffusivity values are obtained as fitting parameters.

\begin{tabular}{|c|c|c|c|c|c|}
\hline $\begin{array}{l}\text { Thickness of } \\
\text { silicon samples } \\
(\mathrm{cm})\end{array}$ & $\begin{array}{l}\text { Mean error in the } \\
\text { calculatedamplitude } \\
\text { (arb. units) }\end{array}$ & $\begin{array}{l}\text { Thermal diffusivity } \\
\text { from amplitude } \\
\left(\mathrm{cm}^{2} / \mathrm{s}\right)\end{array}$ & $\begin{array}{l}\text { Mean error in the } \\
\text { calculated phase } \\
\text { (rad) }\end{array}$ & $\begin{array}{l}\text { Thermal diffusivity } \\
\text { from phase } \\
\left(\mathrm{cm}^{2} / \mathrm{s}\right)\end{array}$ & $\begin{array}{c}\text { Average } \\
\text { thermal diffusivity } \\
\left(\mathrm{cm}^{2} / \mathrm{s}\right)\end{array}$ \\
\hline 0.056 & 0.0015 & 0.8972 & 0.0076 & 0.9181 & 0.9077 \\
\hline 0.06 & 0.0011 & 0.8959 & 0.0470 & 0.8997 & 0.8978 \\
\hline 0.077 & 0.0012 & 0.8971 & 0.0401 & 0.8834 & 0.8903 \\
\hline 0.081 & 0.0021 & 0.9006 & 0.0399 & 0.8824 & 0.8915 \\
\hline 0.094 & 0.0027 & 0.8801 & 0.0021 & 0.9183 & $\begin{array}{c}0.8992 \\
\text { mean }=0.8973\end{array}$ \\
\hline
\end{tabular}




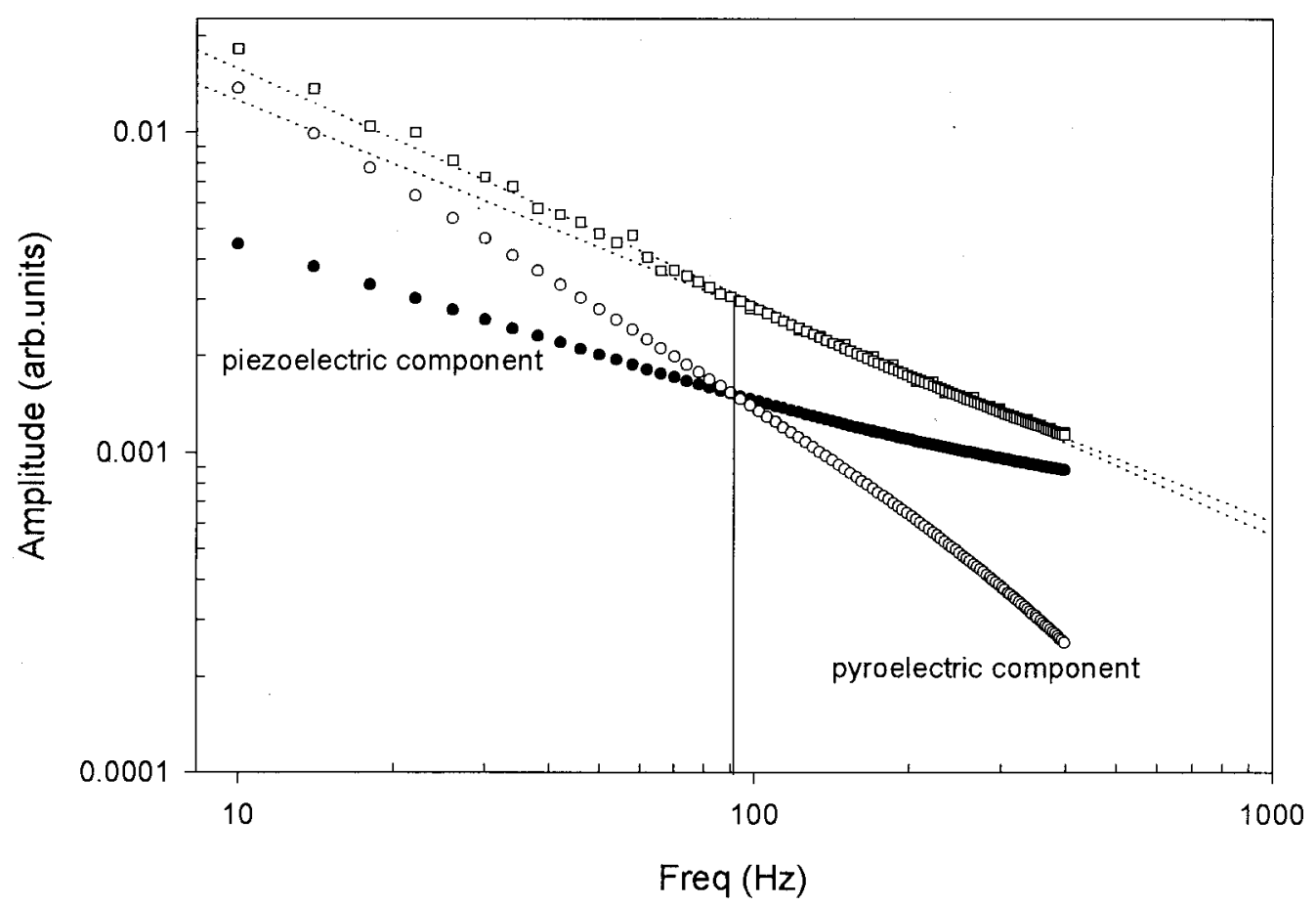

FIG. 5. Comparison between piezoelectric (dots), pyroelectric (circles), and the combined curve (squares) for a silicon sample of thickness $0.056 \mathrm{~cm}$. The crossover of piezoelectric amplitude and pyroelectric amplitude at critical frequency is also shown.

transducer can be used successfully to determine thermal diffusivity of superconducting samples in the transition region. As the transducer can be cut to any shape it is easy to set up the sample within a cryostat for the detection of signals at low temperatures and at low pressures.

The advantage of using a transducer in PA signal detection is that it has a wide frequency response, ranging from a few $\mathrm{Hz}$ to many $\mathrm{MHz}$ and it can be used over a broad range of temperatures and pressures. Since the transducer responds to absorption of radiation by the entire sample rather than within the thermal diffusion length in microphone detection it is very sensitive. In our theory, as we are considering both the piezoelectric photoacoustic phenomenon and pyroelectric photothermal phenomenon, the advantages of both methods are incorporated here and it appears that our method provides a more sensitive approach for measuring the thermal diffusivity.

\section{ACKNOWLEDGMENTS}

The Research Grant Council of Hong Kong and the University of Hong Kong Post Graduate Student Grant sup- ported this work. The major equipment and accessories were supported by the previous UPGC Grant No. 338/025/0005 and University CRGC Grant No. 337/025/003.

${ }^{1}$ C. K. N. Patel and A. C. Tam, Rev. Mod. Phys. 53, 517 (1981).

${ }^{2}$ M. Aravind, P. C. W. Fung, S. Y. Tang, and H. L. Tam, Rev. Sci. Instrum. 67, 1564 (1996).

${ }^{3}$ P. K. Wong, P. C. W. Fung, H. L. Tam, and J. Gao, Phys. Rev. B 51, 523 (1995).

${ }^{4}$ I. V. Blonskij, V. A. Tkhoryk, and M. L. Shendeleva, J. Appl. Phys. 79, 3512 (1996).

${ }^{5}$ W. Jackson and N. M. Amer, J. Appl. Phys. 51, 3343 (1980).

${ }^{6}$ A. Mandelis and M. M. Zver, J. Appl. Phys. 57, 4421 (1985).

${ }^{7}$ C. Christofides, A. Mandelis, K. Ghandi, and R. E. Wagner, Rev. Sci. Instrum. 61, 2360 (1990).

${ }^{8}$ A. Rosencwaig and A. Gersho, J. Appl. Phys. 47, 64 (1976).

${ }^{9}$ S. T. Liu and D. Long, Proc. IEEE 66, 14 (1978).

${ }^{10}$ Y. S. Touloukian, Thermal Diffusivity (IFI/Plenum, New York, 1973).

${ }^{11}$ Handbook of Chemistry and Physics, edited C. D. Hodgman, 44th ed. (Chemical Rubber, Cleveland, OH, 1963).

${ }^{12}$ A. H. Franzan, N. F. Leite, and L. C. M. Miranda, Appl. Phys. A: Solids Surf. 50, 431 (1990).

${ }^{13}$ M. Marinelli, F. Mercuri, U. Zammit, R. Pizzoferrato, and F. Scudieri, Phys. Rev. B 49, 9523 (1994).

${ }^{14}$ S. Bauer and B. Ploss, IEEE Trans. Elec. Insul. 27, 861 (1992). 